\title{
In hot pursuit of the hidden companion of $\eta$ Carinae: an X-ray determination of the wind parameters
}

\author{
Julian M. Pittard \\ Department of Physics and Astronomy, The University of Leeds, \\ Woodhouse Lane, Leeds LS2 9JT, UK \\ Michael F. Corcoran \\ Universities Space Research Association, \\ 7501 Forbes Blvd, Ste 206, Seabrook, MD 20706, USA, and \\ Laboratory for High Energy Astrophysics, \\ NASA Goddard Space Flight Center, Greenbelt, MD 20771, USA
}

\begin{abstract}
We perform X-ray spectral fits to a recently obtained Chandra grating spectrum of $\eta$ Carinae, one of the most massive and powerful stars in the Galaxy and which is strongly suspected to be a colliding wind binary system. The good fit that we obtain gives us further confidence in the binary hypothesis, and we find $\dot{M} \approx 2.5 \times 10^{-4} \mathrm{M}_{\odot} \mathrm{yr}^{-1}$ for the mass loss rate of $\eta$ Car.
\end{abstract}

\section{Introduction}

In recent years, there has been growing evidence that the superluminous star $\eta$ Car (HD 93308, HR 4210) may be a binary (Damineli 1996; Duncan et al. 1995, 1999; Lamers et al. 1998; Corcoran et al. 2000). The presence of an early-type companion means that a powerful wind-wind collision will contribute to the observed emission, and is consistent with observed $\mathrm{X}$-ray and radio variability. A binary interpretation remains somewhat controversial, however, not least because recent HST-STIS spectra did not confirm the predicted variations in the radial velocity of the emission lines (Davidson et al. 2000). To test the binary hypothesis, and to obtain accurate estimates of the wind parameters of each star, we have recently fitted a Chandra grating spectrum against a grid of synthetic spectra calculated from a range of hydrodynamical models of the wind-wind collision (see Pittard \& Corcoran 2002 for further details).

\section{Results}

As $\eta$ Car has a relatively slow wind, the majority of the hard X-ray emission arises from the shocked wind of the companion. In our calculations we fix the wind momentum ratio, $\eta=\left(\dot{M}_{2} v_{\infty_{2}}\right) /\left(\dot{M}_{\infty_{1}} v_{1}\right)$, to 0.2 , and the wind speed of $\eta$ Car to $500 \mathrm{~km} \mathrm{~s}^{-1}$, as suggested from models of the X-ray eclipse. Our best-fit to the data (Figure 1) yields the following wind parameters for the companion: $\dot{M}_{2} \approx 10^{-5} \mathrm{M}_{\odot} \mathrm{yr}^{-1}, v_{\infty_{2}} \approx 3000 \mathrm{~km} \mathrm{~s}^{-1}$. With our previous assumptions this implies that the mass-loss rate of $\eta$ Carinae is $\dot{M}_{1} \approx 2.5 \times 10^{-4} \mathrm{M}_{\odot} \mathrm{yr}^{-1}$. 


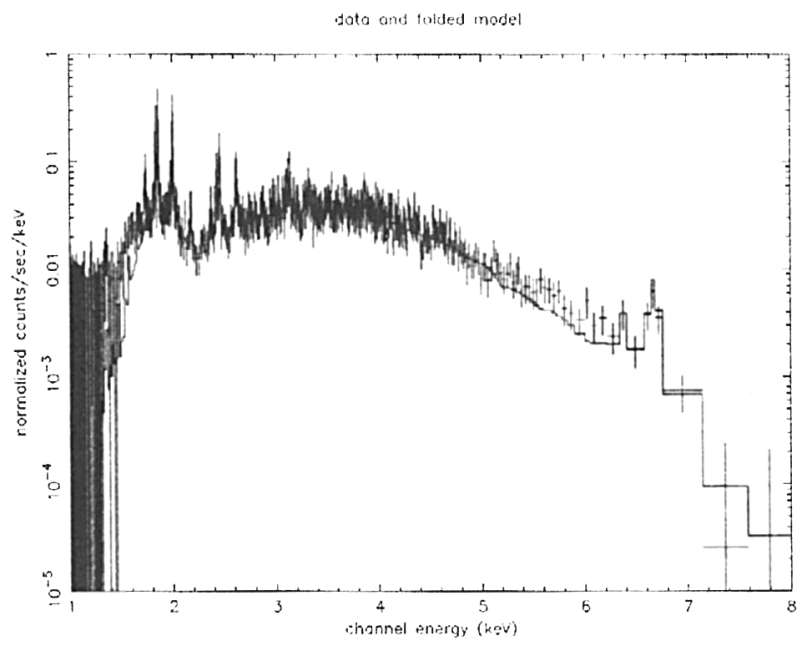

Figure 1. The best fit to the Chandra grating spectrum of $\eta$ Car. The continuum level is matched very well by the interpolated model.

\section{Discussion}

While our analysis does not prove that $\eta$ Car is a binary, we find that the colliding wind emission model naturally provides for the range of ionization seen in the emission lines in the $\mathrm{X}$-ray grating spectrum for reasonable values of the wind parameters. In addition, the X-ray emission from $\eta$ Car is unlike any of the other single stars observed so far at high energies and dispersion and shares some common features with other colliding wind binaries (Corcoran, these Proceedings). The fact that we are able to obtain good fits, with sensible model parameters, gives us further confidence in the binary hypothesis. We further note that unlike in the UV and optical, where fits to the primary are made difficult by significant contamination from the companion star, the hard X-ray emission arises almost exclusively from the shocked wind of the companion. In this sense, our X-ray analysis is entirely complementary to the UV and optical analysis of $\eta$ Car by Hillier et al. (2001).

\section{References}

Corcoran, M.F., Fredericks, A.C., Petre, R., et al. 2000, ApJ 545, 420

Damineli, A. 1996, ApJ (Letters) 460, L49

Davidson, K., Ishibashi, K., Gull, T.R., et al. 2000, ApJ (Letters) 530, L107

Duncan, R.A., White, S.M., Lim, J., et al. 1995, ApJ (Letters) 441, L73

Duncan, R.A., White, S.M., Reynolds, J.E., Lim, J. 1999, in: J.A. Morse, R.M. Humphreys \& A. Damineli (eds.), Eta Carinae at The Millennium, ASP-CS 179, 54

Hillier, D.J., Davidson, K., Ishibashi, K., Gull, T.R. 2001, ApJ 553, 837

Lamers, H., Livio, M., Panagia, N., Walborn, N.R. 1998, ApJ (Letters) 505, L131

Pittard, J.M., Corcoran, M.F. 2002, A\&A 383, 636 